\title{
LACRIMAL SAC RHINOSPORIDIOSIS
}

\author{
Jayant K. Watve,* Rajashri S. Mane, ${ }^{* *}$ Anjana A. Mohite, ${ }^{* *}$ B.C.Patil.***
}

\begin{abstract}
Rhinosporidiosis is a disease caused by the organism Rhinosporidium seeberi, which 2 Wow believed to be a rare aquate protistan parasite of fish. Ocular rhinosporidiosis is worldwide in distribution but relatively nore common in India and Shrilanka. We report a retrospective case record of isolated lacrimal sac rhinosporitiosis in an 8 year male chli from westem Maharashtra. The clinical fearures, management and review of literature is discussed in brief.
\end{abstract}

Keywords: Lacrimal sac Rhinosporidiosis, Endoscopic DCR

\section{INTRODUCTION}

Rhinosporidiosis is a chronic and localised infection of mucous membrane caused by the organism Rhinosporidium Seeberi, which was once thought to be a fungus but is now believed to be a rare aquatic protistan parasite of fish. ${ }^{1}$ First described in 1900 by Guillermo Seeber, it affects the nasal cavity, ocular adenexa, throat and genitalia in both sexes. Though ocular sporidiosis is world wide in distribution, it is seen relatively more prevalent in southern India, Srilanka, and South-east Asia.

Here we report a retrospective case study of an 8-year-old boy diagnosed as dacryocystitis. A soft, pink, polypoidal mass from the lacrimal sac on histopathological evaluation was diagnosed as rhinosporidiosis. The child is well post operatively and asymptomatic till date.

\section{CASE REPORT}

In Feb. 2005, an 8-year old male child was referred by the Department of Ophthalmology for endonasal endoscopic dacryocystorhinostomy (DCR), for complaints of watering from Right eye and swelling near medial canthus since 2 months. The swelling increased in size to a peanut with continuous epiphora, associated with purulent discharge at times. External examination of right eye revealed a soft globular, non-tender swelling near right medial canthus. Pressure over the swelling showed regurgitation of fluid from the puncta, indicating lacrimal sac pathology. The vision and ocular movements were normal. The nose, throat and left ocular examination revealed no abnormality. CT scan and dacryocystography were not performed as the patients economical condition did not permit.

After routine investigations and anesthesia fitness, child was posted for endonasal DCR. The sac was opened up after making a wide bone window. Intraoperative bleeding was little more than expected, which was thought to be due to GA. A small polypoidal mass popped out after opening of the sac. This appeared little unusual than the muco purulent discharge, which is usually seen. The soft, bleeding, polypoidal mass, was excised and sent for histopathological examination. Surgery was uneventful. Intraoperative sac syringing revealed patency of the nasolacrimal system.

The histopathology was reported as Rhinosporidiosis of lacrimal sac. Sac syringing was performed for 2 weeks. The child was then put on alternate day $100 \mathrm{mg}$. of diaminodiphenylsulfone (DDS) (Dapsone), and was advised to continue the same for a year. Regular follow-ups have shown patent and disease free lacrimal sac till date.

\section{DISCUSSION}

Rhinosporidiosis is presumably a waterborne disease and generally occurs after swimming in stagnant freshwater ponds, lakes, or rivers, but is also suspected to occur from dust or air. Though world wide in distribution, $88 \%$ of cases are reported from India and Srilanka. ${ }^{2}$ Primary ocular sporidiosis was reported in $10 \%$ cases, of which, $7.32 \%$ involved the lacrimal sac. The disease was seen most frequently in young children between eight and ten years of age. Males were infected more than females. $(2.5: 1){ }^{3}$ Our case was an 8 -year-old male child, who gave history of swimming in a stagnant water container.

The lacrimal sac is usually affected by infection ascending from the nose. The infection then has tendency to spread diffusely underneath the skin. ${ }^{4}$ However, in this patient, there was isolated involvement of nasolacrimal sac without any nasal or conjunctival component.

In nasolacrimal rhinosporidiosis, spread of infection is pericanalicular and perisacular, and that is why epiphora is not a common symptom. ${ }^{4}$ In contrast our case patient had initially intermittent and later continuous epiphora, purulent at times, suggestive of intracanalicular and intra sacular spread. Although dacryocystectomy has been described as a modality to treat lacrimal sac sporidiosis, the same was not done in our case, since ours was a retrospective diagnosis.

As possibility of contamination of nose can not be excluded, this child is kept on regular follow up and oral DDS 100-mg alternate day for a year. The administration of DDS (Dapsone) reduces rate of postoperative recurrence and this is attributed to an arrest of maturation of the spores and accentuated grannulomatous response with fibrosis after Dapsone therapy. ${ }^{5}$

Though the diagnosis rests with the ophthalmologists, ENT surgeons are well versed with the use of endoscope and endonasal anatomy of lacrimal sac. The Lacrimal sac pathology, whether 


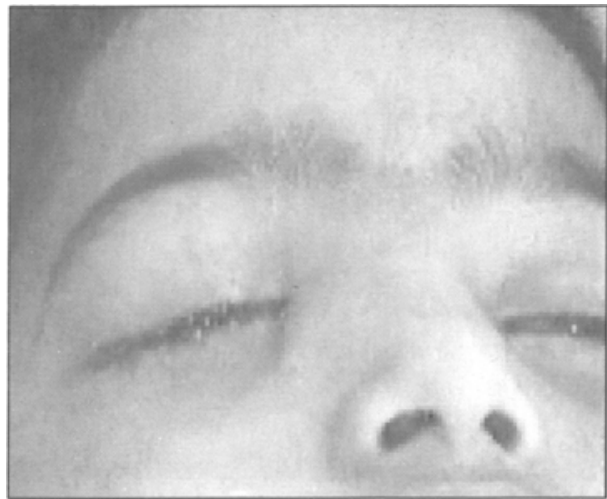

Fig I : Clinical photograph of the child with swelling near Rt. medical canthus

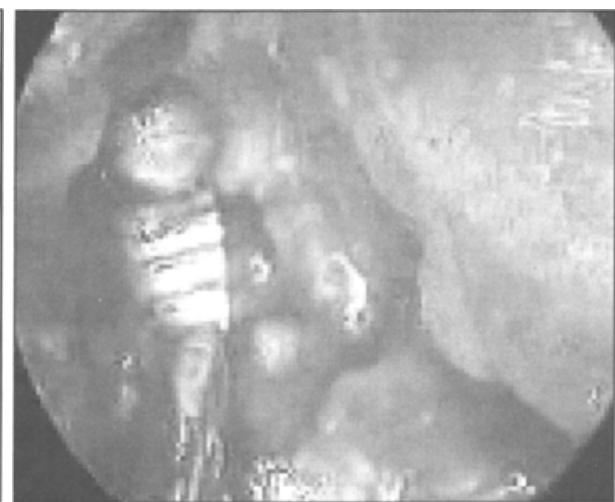

Fig II : Intra operative photograph showing polypoidal mass from the lacrimal sac

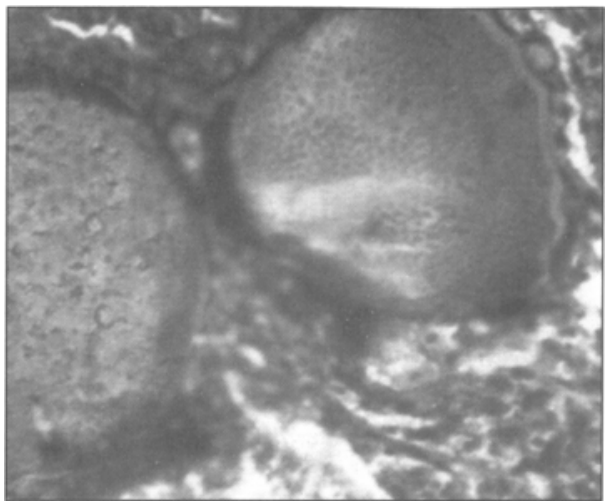

Fig III : Histopathology of the lesion stained with hematoxylin and esosin, under high power view, showing sporangia. dacryocystitis or otherwise, is better dealt with the endoscopic approach for its direct exposure and minimal discomfort to the patient. This avoids external scar and seeding at external incision site. $^{6}$

\section{CONCLUSION}

In all cases of lacrimal sac pathology, one needs to keep a clinical diagnosis of rhinosporidiosis in mind. Although dacryocystectomy has been described as the treatment of choice, an endonasal DCR with excision of the lesion, followed by postoperative dapsone therapy for a year, could suffice. However, long term follow up and more number of case studies are necessary to establish efficacy of this modality of management.

\section{REFERENCES}

1 Ahluwalia, KB. "Causative agent of rhinosporidiosis." Journal of Clinical Microbiology. January 2001. 39(1): 413-5.

2 Karunaratne WAE; Rhinosporidiosis in Man. Athlone, University of London, 1964
3 Shrestha SP, Hennig A, Parija SC: Prevalence of rhinosporidiosis of the eye and its adenexa in Nepal.: American Journal Of Tropical Medicine And Hygiene 59(2) 1998, pp. 231-234.

4. Kameshwaran S.: ENT Diseases in a Tropical Environment (ed.2). Chennai MERF Pvt. Ltd., 1999.

5. Job A, Venkateswaran S, Mathan M, et. al: Medical therapy of rhinosporidiosis with Dapsone. J. Laryngol Otol 107:809-812, 1993.

6. Nerurkar et.al; Lacrimal sac rhinosporidiosis: a case report: American Journal of Otolaryngology 25(6):423-5, 2004 Nov-Dec.

Address for correspondence:

Dr. Jayant K. Watve Asso. Prof., Dept. Of ENT Dr. D.Y. Patil Medical College \& Hospital, Kadamwadi, Kolhapur, Maharashtra

\title{
NASOPHARYNGEAL CARCINOMA PRESENTING AS GRADENIGO'S SYNDROME
}

\author{
Avik Kumar Jana, ${ }^{*}$ Abhishek Jaswal,* Biswajit Sikder, ** Utpal Jana, ** Tapan Kumar Nandi.***
}

\begin{abstract}
Nasopharyngeal carcinoma usually has an insidious onset and non-specific features in the initial stages. This makes early diagnosis difficult. The most usual presenting features are cervical bmphadenopathy, otological symptoms (serous otitis media) and involvement of adjacent cranial nerves. We report a case of Gradenigo's syndrome in a patient of undifferentiated nasopharyngeal carcinoma.
\end{abstract}

Keywords: Nasopharyngeal carcinoma, Gradenigo's syndrome

\section{INTRODUCTION}

Classic gradenigo syndrome with triad of sixth nerve palsy, pain in the distribution of the fifth nerve, and otitis media is very rare entity these days. Though generally caused by otitis media and mastoiditis in the region of the apex of the petrous temporal bone, in recent times, other causes have been recognized for instance nasopharyngeal malignancies, meningioma, fractures, and 'T'cell

${ }^{*}$ Pgt, **Assistant professors, ***Professor, Department of Otorhinolaryngology, Calcutta National Medical College, Avik Kumar Jana, ClO Asoke Kr Jana, Model Town, Garia, Balia, Kolkata - 84, W.B, India.

Indian Journal of Otolaryngology and Head and Neck Surgery Vol. 58, No. 4, October - December 2006 